\title{
Structure of an ALS/MSP-related D290V-mutation-containing hexapeptide in hnRNPA2 low-complexity domain reveals differences from its corresponding wild type peptide
}

\author{
D Eisenberg ${ }^{1}$, Q Cao², J Lu², M Hughes ${ }^{2}$, M Sawaya ${ }^{2}$, D Boyer², D Cascio', D Eisenberg ${ }^{1}$ \\ ${ }^{1}$ Univ Of California Los Angeles, Los Angeles, CA, ${ }^{2}$ UCLA-DOE Institute, Los Angeles, CA \\ david@mbi.ucla.edu
}

Heterogeneous nuclear ribonucleoprotein A2 (hnRNPA2) is an RNA-binding protein involved in RNA transport and stabilization through a membraneless organelle named stress granule. Disruption of stress granules dynamic is associated with neurodegenerative diseases like amyotrophic lateral sclerosis (ALS) and multisystem proteinopathy (MSP). HnRNPA2 contains two RNA recognition motifs and one low-complexity region (LCD). A familial ALS/MSP-related mutation (D290V) within the hnRNPA2 LCD has been identified in previous studies that show evidence of it exacerbating the hnRNPA2 LCD's tendency to assemble into fibrils. We determined the crystal structure of a key hexapeptide (GNYNVF) containing this single point mutation and compared it biochemically to the wild type hexapeptide (GNYNDF). The mutant peptide forms a "steric zipper" motif and the sidechains of the two $\beta$-sheets mate tightly with each other in a dry interface. As expected, the mutant peptide is energetically stable and forms a homo-zipper. We then seek to characterize the differences between the wild type and the mutant peptides by electron microscopy. We shook both peptide solutions for a week at room temperature. Due to its energetic stability, mutant peptide forms white precipitates which are composed of wide thick crystals. On the contrary, the wild type peptide forms a hydrogel, which to our knowledge is the shortest segment that forms a hydrogel, and TEM images indicate that the hydrogel is composed of thin fibrils. The wild type peptide exhibits similar physical behavior as the full-length hnRNPA2 LCD, being able to form a hydrogel that is composed of amyloid-like fibrils. This result suggests that the mutant peptide GNYNVF with the pathogenic mutation forms a more stable structure than the wild type peptide GNYNDF, and converts a hydrogel-promoting segment into an aggregation-promoting segment.

Acta Cryst. (2020). A76, a147 